\title{
Las clases prácticas de Fisiología Humana y el trabajo previo del estudiante utilizando la plataforma MOODLE: un estudio a pie de aula.
}

Sergio Rius-Pérez ${ }^{a}$, Isabel Torres-Cuevas ${ }^{a}$, Eva Tamayo ${ }^{a}$ y Salvador Pérez-Garrido ${ }^{a}$

a'Departamento de Fisiología, Facultad de Farmacia, Universidad de Valencia. Sergio.Rius@uv.es; torrescuevasisabel@gmail.com; eva.tamayo@uv.es; Salvador.Pérez-Garrido@uv.es.

\section{\$EWWDW}

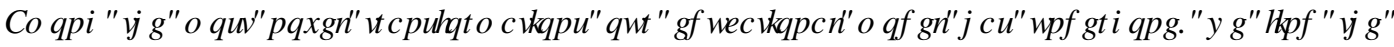

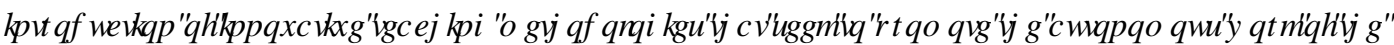

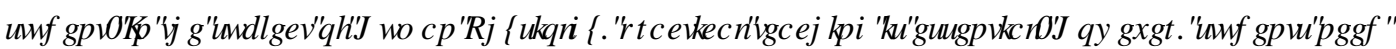

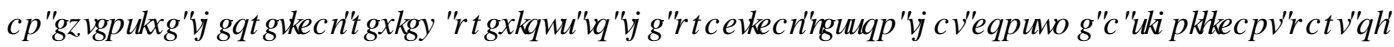

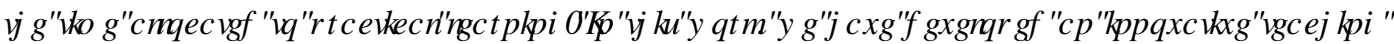

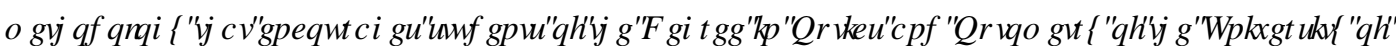

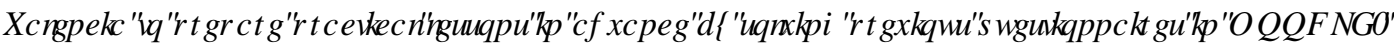

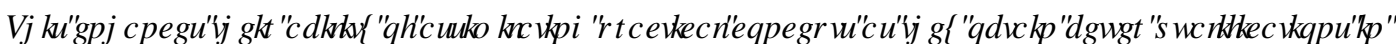

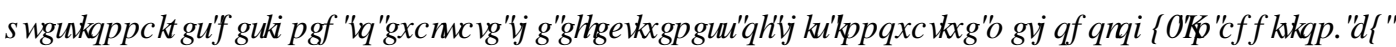

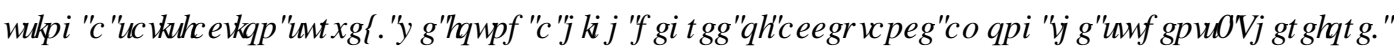

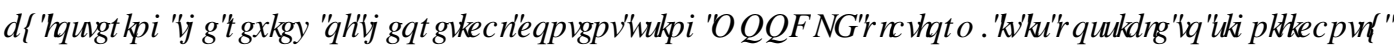

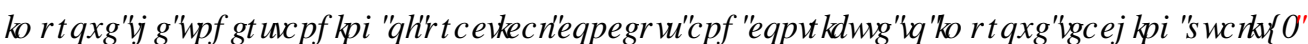

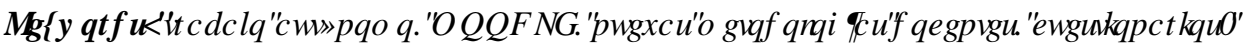

\section{HXP HQ]}

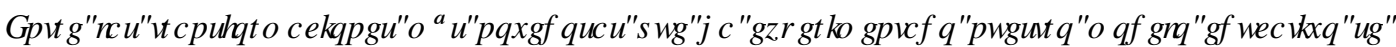

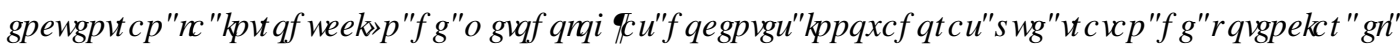

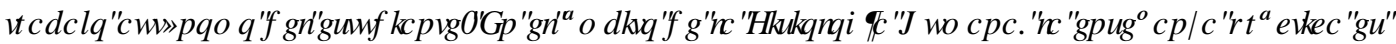

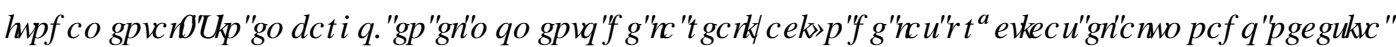

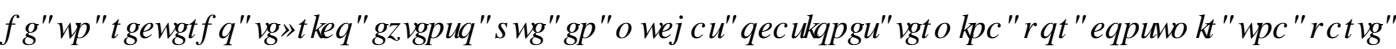

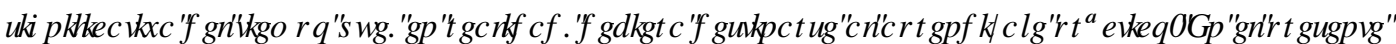

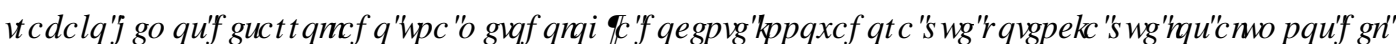

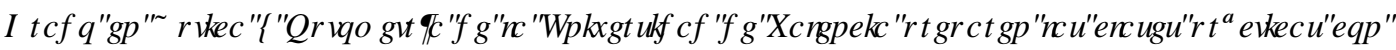

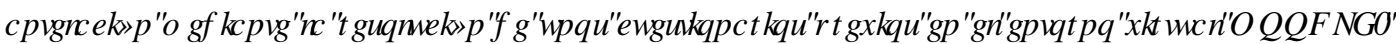

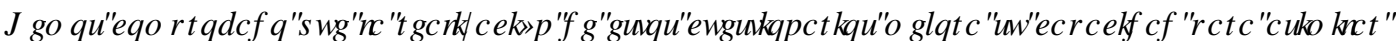
IRVFRQUAQIGRVSU FUFRVI DITXHREUHQHQP HVRHVIFDOIIFDFIRQHVHQXQRVFXHMRQDURVISRUQSU FUFD

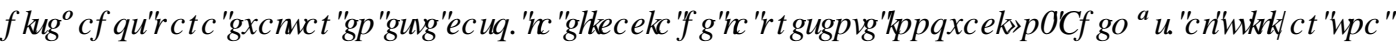

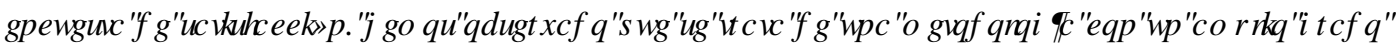

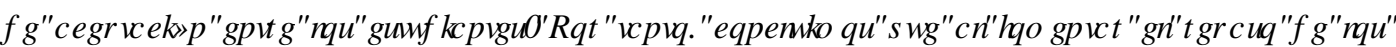

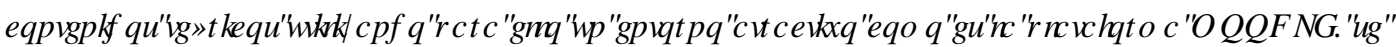

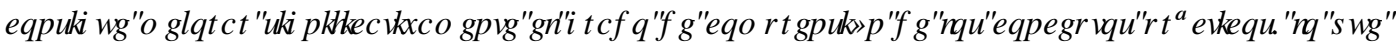

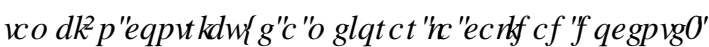

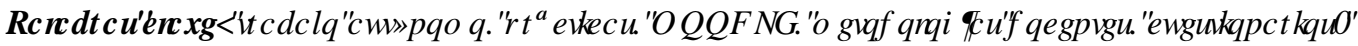




\section{Introducción}

La implantación del Espacio Europeo de Educación Superior (EEES) en la universidad española ha requerido de cambios profundos en los métodos docentes y en las formas de trabajo de los estudiantes. Entre las transformaciones más novedosas que ha experimentado nuestro modelo educativo se encuentran la introducción de nuevas metodologías docentes innovadoras que tratan de potenciar el trabajo autónomo del estudiante y las capacidades de éstos para crear sus propios procesos de aprendizaje (RodríguezIzquierdo, 2014; Prudencia Gutiérrez HWDO, 2011).

Desde un punto de vista cognitivo, la autonomía en el aprendizaje requiere que el estudiante adquiera un grado significativo de responsabilidad sobre el propio proceso de aprendizaje lo que le convierte en parte activa del mismo (Rué, 2009). Se trata, en definitiva, de un modelo de aprendizaje basado en una filosofía constructivista donde es el alumno el que, con su esfuerzo y su trabajo continuo, adquiere los conocimientos y desarrolla las competencias y habilidades que en el futuro le garantizarán el éxito académico y profesional (Rodríguez-Izquierdo, 2014; Tejada y López, 2012). Sin embargo, la correcta implantación de este modelo hace necesario desarrollar nuevas metodologías docentes que favorezcan la autonomía de los estudiantes, así como impulsar el uso de herramientas y entornos virtuales dedicados, en esencia, a arraigar en el estudiante la idea de que el conocimiento con el que se encuentra no es algo dado, sino que requiere de su participación activa para generarlo e interiorizarlo.

En el ámbito de la Fisiología Humana, la enseñanza práctica es fundamental para conseguir un aprendizaje adecuado del estudiante en esta materia. La enseñanza práctica de la Fisiología implica una enseñanza participativa, de trabajo práctico, donde se fomenta el espíritu crítico del estudiante, profundamente distanciada del mero aprendizaje memorístico en el que el estudiante desempeña un papel eminentemente pasivo (García, 2013; Patrick, 2011). Además, constituye un complemento esencial de la clase teórica ya que ayuda a visualizar mejor determinados conceptos que, por su complejidad, resultarían de muy difícil comprensión sin una experiencia práctica (Olmos HWDO, 2014). Sin embargo, en muchas ocasiones los contenidos teóricos y prácticos no se imparten de manera síncrona a lo largo del programa de la asignatura debido a limitaciones de diversa casuística que pueden incluir desde problemas de coordinación horaria hasta dificultades en la disponibilidad de espacios, entre otras. Es por ello por lo que, en muchas ocasiones, para garantizar una adecuada comprensión de los contenidos prácticos, se requiere que el estudiante realice un trabajo autónomo previo basado en una revisión exhaustiva de los contenidos teóricos de la asignatura que posteriormente aplicará en las clases prácticas. Sin embargo, la escasez de material docente que cubra estas necesidades, así como la ausencia de metodologías adecuadas que apoyen y dirijan el trabajo autónomo del estudiante hacen que este trabajo previo habitualmente no se lleve a cabo. En consecuencia, en el momento de la realización de las prácticas el alumnado necesita de un recuerdo teórico extenso que en muchas ocasiones termina por consumir una parte significativa del tiempo que, en realidad, debiera destinarse al aprendizaje experiencial. Además, este recuerdo teórico suele impartirse siguiendo el modelo de clase magistral, muy alejado de la filosofía constructivista del aprendizaje. Nos encontramos pues ante un escenario en el que la innovación educativa, entendida ésta no solamente como la introducción de nuevas metodologías sino también como la mejora de las ya existentes (Zabalza, 2003-4), puede contribuir de forma significativa a mejorar y preservar el aprendizaje experiencial, un valor en sí mismo en la asignatura de Fisiología Humana que rara vez puede desarrollarse fuera del contexto de las clases prácticas.

Teniendo en cuenta todo lo expuesto y dada nuestra experiencia en la enseñanza práctica de la Fisiología Humana, hemos desarrollado una metodología docente innovadora que potencia que los alumnos del Grado en Óptica y Optometría de la Universidad de Valencia preparen las clases prácticas con antelación mediante la resolución de unos cuestionarios previos en el entorno virtual MOODLE. De este modo, no solamente 
se fomenta el trabajo autónomo del alumno, sino que se incorpora el uso de nuevas tecnologías educativas en el aprendizaje práctico de la Fisiología Humana.

\section{Objetivos}

Los objetivos del presente trabajo fueron:

1. Desarrollar, mediante el uso de la plataforma virtual MOODLE, unos cuestionarios preparatorios de las prácticas de la asignatura de Fisiología Humana y Ocular del Grado en Óptica y Optometría de la Universidad de Valencia.

2. Demostrar que fomentar el trabajo autónomo del estudiante con antelación a las prácticas mediante la resolución de cuestionarios en la plataforma virtual MOODLE mejora la comprensión de los contenidos prácticos de esta asignatura.

3. Conocer la percepción de los estudiantes respecto de la utilización de cuestionarios en la plataforma virtual MOODLE para la preparación de las prácticas de esta asignatura.

\section{Desarrollo de la innovación}

La innovación desarrollada en el presente trabajo fue dirigida a los alumnos de primer curso del Grado en Óptica y Optometría de la Universidad de Valencia en la asignatura de Fisiología Humana y Ocular, durante el segundo cuatrimestre del curso 2020-2021. Se trata de una asignatura de 9 ECTS que se ocupa del estudio del funcionamiento de los órganos, aparatos y sistemas que componen el organismo humano. Se estudia su funcionamiento desde el nivel molecular y celular hasta el nivel integral de la persona, la interrelación existente entre los sistemas y con el medio externo, así como los mecanismos de regulación e integración funcional que hacen posible la vida. Si bien la asignatura hace especial énfasis en el estudio de la función visual, al tratarse de una materia de formación básica, uno de sus objetivos fundamentales es proporcionar al alumno una base biomédica útil respecto del comportamiento de todos los sistemas fisiológicos del organismo humano, no solamente los relacionados con la visión, que le ayude a comprender la integración de la función visual en toda la fisiología humana. Por ello, la asignatura se divide en una parte general y otra específica que representan un $60 \%$ y un $40 \%$ del programa, respectivamente. En la parte general las clases teóricas se complementan con clases prácticas, lo que permite cubrir las necesidades de aprendizaje experiencial que requiere esta materia. Es en el desarrollo de estas clases prácticas donde se ha llevado a cabo la presente innovación, en concreto, en las prácticas que versan sobre las siguientes temáticas: potencial acción, sensibilidad somática, audiometría y auscultación cardíaca y presión arterial.

\subsection{Participantes}

La muestra estuvo constituida por 48 estudiantes de primer curso de la asignatura de Fisiología Humana y Ocular del Grado en Óptica y Optometría de la Universidad de Valencia. De los participantes el 74,41\% eran mujeres y el 25,58\% varones. Sus edades oscilaban entre los 18 y 22 años, siendo la media de edad de 19 años. La participación en el estudio fue voluntaria y se comunicó a los participantes que al finalizar la investigación se les informaría sobre los resultados y conclusiones del estudio.

\subsection{Implementación de la innovación}

Para el desarrollo de la innovación los estudiantes pertenecientes a dos grupos de 24 alumnos de la asignatura Fisiología Humana y Ocular fueron divididos a su vez en dos subgrupos de 12 alumnos cada uno. En cada una de las prácticas, a los alumnos de uno de los dos subgrupos se les solicitó que respondieran un cuestionario alojado en la plataforma virtual MOODLE dos días antes de la fecha de realización de la

(c)) BY-NC-ND 2021, Universitat Politècnica de València

CRQJHMR, Q5 HGHपQTI 
práctica. El otro subgrupo realizó la práctica sin responder a ningún cuestionario previo. A lo largo de la realización de las cuatro prácticas se fue intercambiando el rol de cada subgrupo, respondiendo al cuestionario previo unos subgrupos y otros no, de forma alterna, tal y como se muestra en la Tabla 2. Una vez finalizada la clase práctica todos los alumnos, independientemente del subgrupo al que pertenecieran, realizaron un cuestionario (cuestionario post-práctica) alojado también en la plataforma virtual MOODLE que evaluaba la consecución de los objetivos previstos para cada práctica.

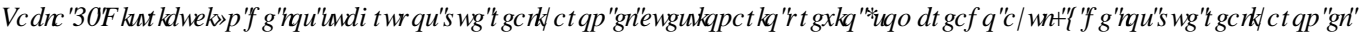
FXHMURDURISRUASU FUFDDVRP EUHDRTYHGHW

\begin{tabular}{|c|c|c|c|c|c|c|c|c|c|c|c|c|c|c|c|c|}
\hline \multirow[b]{3}{*}{ Práctica } & \multicolumn{8}{|c|}{ Grupo $1(n=24)$} & \multicolumn{8}{|c|}{ Grupo $2(n=24)$} \\
\hline & \multicolumn{4}{|c|}{$\begin{array}{l}\text { Subgrupo } 1 \\
\qquad(\mathrm{n}=12)\end{array}$} & \multicolumn{4}{|c|}{$\begin{array}{c}\text { Subgrupo } 2 \\
(n=12)\end{array}$} & \multicolumn{4}{|c|}{$\begin{array}{l}\text { Subgrupo } 1 \\
(n=12)\end{array}$} & \multicolumn{4}{|c|}{$\begin{array}{c}\text { Subgrupo } 2 \\
(n=12)\end{array}$} \\
\hline & 1 & 2 & 3 & 4 & 1 & 2 & 3 & 4 & 1 & 2 & 3 & 4 & 1 & 2 & 3 & 4 \\
\hline Cuestionario previo & & & & & & & & & & & & & & & & \\
\hline Cuestionario post-práctica & & & & & & & & & & & & & & & & \\
\hline
\end{tabular}

Prácticas: 1 (potencial de acción); 2 (sensibilidad somática); 3 (audiometría); 4 (auscultación cardíaca y presión arterial)

Tanto los cuestionarios previos como los cuestionarios post-práctica constaban de cinco preguntas de las cuales solo una de ellas era correcta. Mientras que para los cuestionarios post-práctica se estableció un límite de tiempo de 15 minutos para su contestación, para los cuestionarios pre-práctica no se impuso ningún límite de tiempo ya que se presentaron a los estudiantes como herramienta de repaso de los conceptos teóricos. Una vez finalizado el cuestionario, gracias al entorno virtual que proporciona MOODLE, los estudiantes pudieron comprobar si sus respuestas habían sido o no correctas. En cuanto al contenido de los cuestionarios, mientras que los test previos trataron de cubrir aspectos teóricos ya explicados con anterioridad pero que convenía recordar para asimilar mejor la sesión práctica, los test post se orientaron con la finalidad de evaluar el grado de comprensión de aquellos conceptos novedosos que habían sido expuestos en la propia sesión práctica. La temática de unos y otros cuestionarios se resume en la Tabla 2.

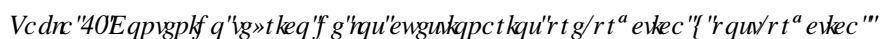

\begin{tabular}{|c|c|c|}
\hline PRÁCTICA & Cuestionario pre-práctica & Cuestionario post-práctica \\
\hline Potencial de acción & $\begin{array}{l}\text { - Transporte de iones } \\
\text { - Concepto de potencial de membrana, } \\
\text { potencial de acción, periodo } \\
\text { refractario relativo y absoluto. }\end{array}$ & $\begin{array}{l}\text { - Curva de intensidad-tiempo: concepto de } \\
\text { reobase y cronaxia } \\
\text { - Cálculo de la duración del periodo } \\
\text { refractario relativo y absoluto }\end{array}$ \\
\hline Sensibilidad somática & $\begin{array}{l}\text { Elementos de la percepción sensorial: } \\
\text { recpetor, efector y centro nervioso } \\
\text { - Tipos de receptores y mecanismos de } \\
\text { adaptación. }\end{array}$ & $\begin{array}{l}\text { - Percepción de sensaciones táctiles } \\
\text { - Discriminación espacial } \\
\text { - Campos receptivos }\end{array}$ \\
\hline Audiometría & $\begin{array}{l}\text { - Frecuencia e intensidad sonora } \\
\text { - Discriminación de sonidos en el oído } \\
\text { interno }\end{array}$ & $\begin{array}{l}\text { - Interpretación de las pruebas de Rinne y } \\
\text { Weber } \\
\text { - Interpretación de una audiometría }\end{array}$ \\
\hline $\begin{array}{c}\text { Auscultación cardíaca y } \\
\text { presión arterial }\end{array}$ & $\begin{array}{l}\text { - Regulación del fujo sanguíneo } \\
\text { - Ciclo cardiaco }\end{array}$ & $\begin{array}{l}\text { - Ruidos cardíacos } \\
\text { - Interrpretación de los cambios en la } \\
\text { presión arterial }\end{array}$ \\
\hline
\end{tabular}


Finalmente, una vez terminadas las cuatro prácticas y con el objetivo de conocer la percepción de los estudiantes de la presente innovación, se les facilitó una encuesta en la que debían valorar una serie de afirmaciones del 1 al 5 en función de su grado de acuerdo o desacuerdo. Los ítems planteados en esta encuesta se detallan en la Tabla 3.

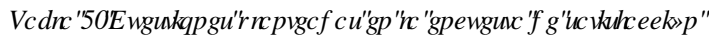

\begin{tabular}{l||l}
\hline $\mathbf{1}$ & Las preguntas planteadas en los cuestionarios previos se adecúan al contenido de las prácticas \\
\hline $\mathbf{2}$ & La realización de los cuestionarios previos me ha ayudado a entender mejor la práctica \\
\hline $\mathbf{3}$ & $\begin{array}{l}\text { He notado diferencia al enfrentarme a las prácticas los días que me había tocado hacer los } \\
\text { cuestionarios previos respecto de los que no. }\end{array}$ \\
\hline $\mathbf{4}$ & La realización de los cuestionarios pre y post-práctica me ayudarán a superar el examen de prácticas \\
\hline $\mathbf{5}$ & Valora la actividad del 1 al 5 \\
\hline
\end{tabular}

\subsection{Análisis estadístico}

Para el análisis de datos se utilizó el programa estadístico SPSS 23. Para determinar si existían diferencias estadísticamente significativas entre las medias de los grupos de estudio se utilizó el estadístico W6WGHW La prueba Chi-cuadrado se utilizó para determinar si existía relación entre los porcentajes de acierto en los tests y la metodología empleada. Los análisis de correlación se llevaron a cabo utilizando el método de Pearson. Se consideraron diferencias estadísticamente significativas si $\mathrm{p}<0.05$.

\section{Resultados}

En primer lugar, se comprobó que en aquellos subgrupos que realizaron el test pre-práctica la media de las calificaciones obtenidas en cada uno de los cuestionarios no fue estadísticamente diferente de la que obtuvieron en el test post-práctica (Tabla 4). De hecho, al analizar las calificaciones de cada alumno, con independencia de la sesión práctica, se observó que existía una correlación estadísticamente significativa entre la calificación que el alumno obtenía en el pre-test y la que obtenía en el post-test $(r=0,256 ; p=0,02)$.

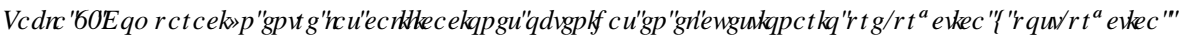

\begin{tabular}{c|c|c}
\hline \multirow{2}{*}{ PRÁCTICA } & \multicolumn{2}{c}{ CALIFICACIONES } \\
\cline { 2 - 3 } & $\begin{array}{c}\text { Cuestionario pre- } \\
\text { práctica }\end{array}$ & $\begin{array}{c}\text { Cuestionario post- } \\
\text { práctica }\end{array}$ \\
\hline \hline Potencial de acción & $6,94 \pm 1,74$ & $7,29 \pm 2,23$ \\
\hline \hline Sensibilidad somática & $7,81 \pm 2,09$ & $8,57 \pm 2,11$ \\
\hline \hline Audiometría & $6,15 \pm 2,13$ & $5,39 \pm 2,37$ \\
\hline \hline Auscultación cardíaca y presión arterial & $6,56 \pm 2,64$ & $6,74 \pm 1,52$ \\
\hline
\end{tabular}

Una vez comprobadas que las calificaciones en el test pre-práctica y post-práctica eran comparables y que incluso se correlacionaban, evaluamos la distribución de aciertos en el test post-práctica en función de si el subgrupo había realizado previamente el pre-test o no. En las prácticas de potencial de acción, audiometría 
y auscultación cardíaca y presión arterial, se observó una dependencia estadísticamente significativa entre las proporciones de aciertos y la metodología empleada, si bien no pudo hallarse significancia estadística en la práctica de sensibilidad somática (Fig.1). Se trata, ésta última, de una temática en la que los estudiantes fueron capaces de alcanzar calificaciones más elevadas, tanto en los cuestionarios pre-práctica como postpráctica (Tabla 4). Es probable que la sencillez de los contenidos de esta práctica (significativamente menos complejos que aquellos desarrollados en el resto de las prácticas) tienda a anular el efecto de la metodología empleada. De hecho, un 47,61\% de los estudiantes (independientemente de haber realizar el cuestionario previo o no) fue capaz de responder correctamente la totalidad de este test post-práctica (Fig. 1B), porcentaje que contrasta con el obtenido en el resto de las prácticas (Fig. 1A, 1C, 1D).

A

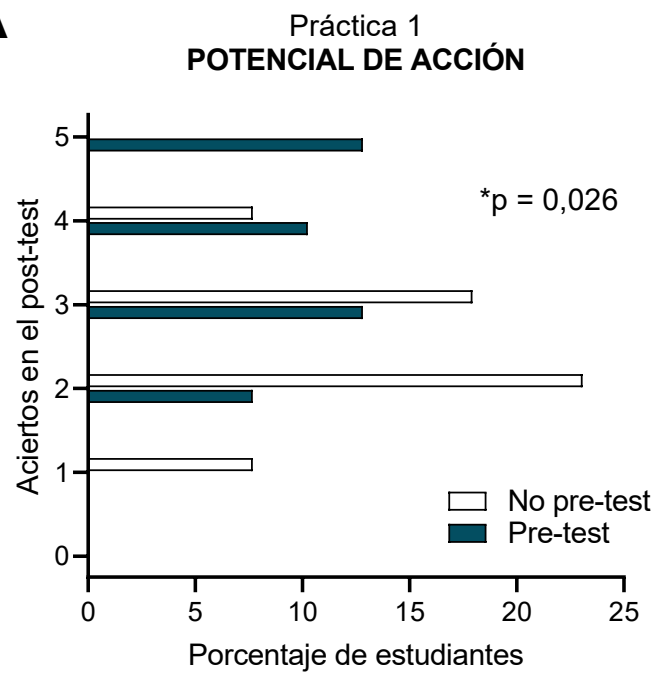

C

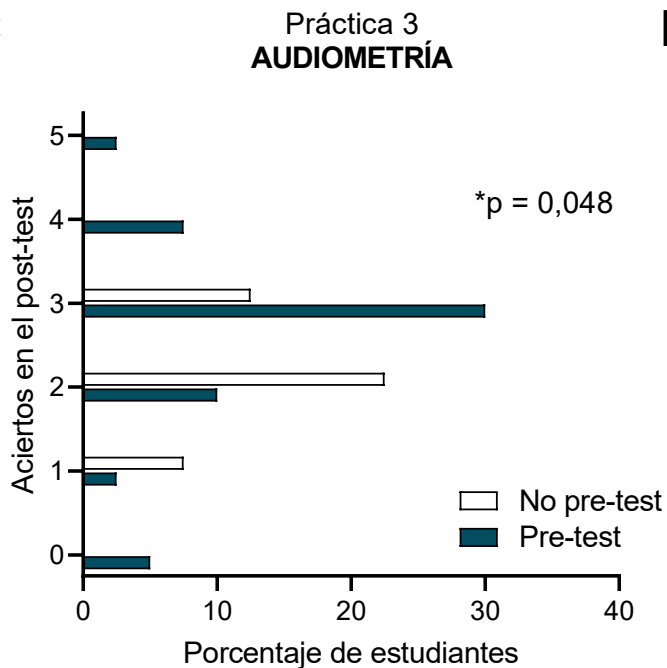

B

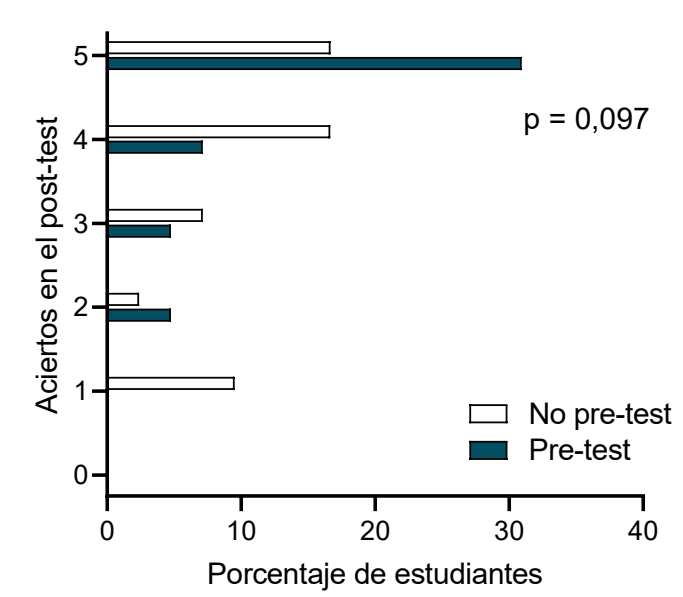

D

Práctica 4

AUSCULTACIÓN CARDÍACA

Y PRESIÓN ARTERIAL

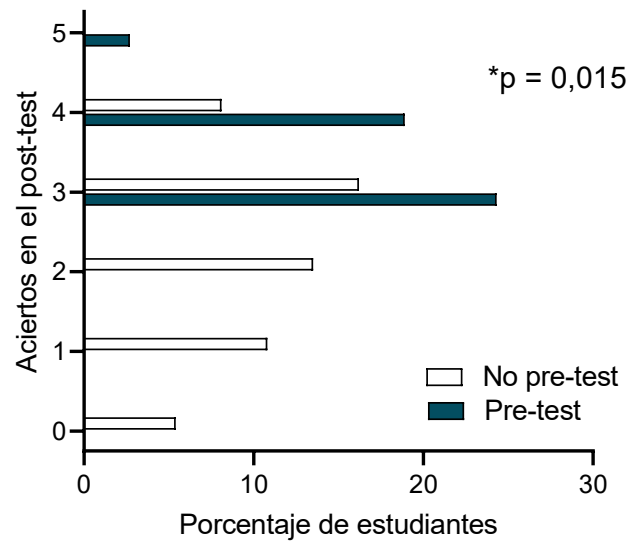

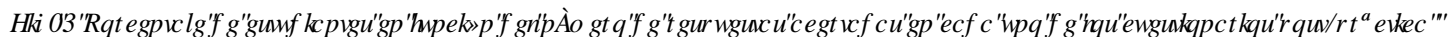

El análisis detallado de la Figura 1 nos permite observar cómo en la práctica de potencial de acción (Fig. 1A), todos los alumnos que fueron capaces de acertar la totalidad de las preguntas del cuestionario (el $12,82 \%$ de los estudiantes) habían realizado el test previo. Sin embargo, los alumnos que únicamente respondieron correctamente a una pregunta (el 7,69\% de los estudiantes) pertenecía al subgrupo que no realizó el test pre-práctica. En la práctica de audiometría (Fig. 1C) ocurrió algo similar: todos los estudiantes capaces de responder a más de cuatro preguntas correctamente (el 10\% de los estudiantes) habían realizado 
el cuestionario previo. En lo que respecta a la práctica de auscultación cardíaca y presión arterial (Fig. 1D), ningún alumno que había respondido al pre-test obtuvo menos de 3 aciertos.

De acuerdo con los datos mostrados en la Figura 1, se comprobó que la media de las calificaciones obtenidas en los test post-práctica era significativamente mayor en los subgrupos donde se había llevado a cabo la innovación (Tabla 5). De hecho, en las tres prácticas en las que la metodología innovadora mostró un efecto positivo, la no realización del cuestionario pre-práctica supuso que la media de las calificaciones obtenidas ni siquiera alcanzara el aprobado. Tanto es así que, al tomar en conjunto las calificaciones de las cuatro prácticas, comprobamos que la realización del test previo supuso que la nota global de los tests post-práctica mejorara en 1,83 puntos de media (Tabla 5).

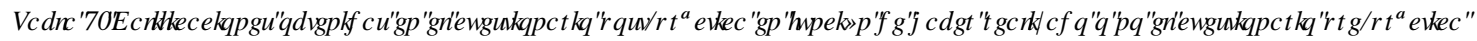

\begin{tabular}{|c|c|c|}
\hline \multirow{2}{*}{ PRÁCTICA } & \multicolumn{2}{|c|}{ CALIFICACIONES } \\
\hline & Subgrupos NO pre-test & Subgrupos pre-test \\
\hline Potencial de acción & $4,91 \pm 1,82$ & $7,29 \pm 2,23 * *$ \\
\hline Sensibilidad somática & $7,09 \pm 2,94$ & $8,57 \pm 2,11$ \\
\hline Audiometría & $4,24 \pm 1,39$ & $5,39 \pm 2,37 *$ \\
\hline Auscultación cardíaca y presión arterial & $4,40 \pm 2,48$ & $6,74 \pm 1,52 * *$ \\
\hline PROMEDIO & $5,16 \pm 2,15$ & $6,99 \pm 2,05 * *$ \\
\hline
\end{tabular}

Finalmente, mediante el análisis de los resultados de la encuesta de satisfacción se comprobó que un 59,08\% de los estudiantes se mostraba bastante de acuerdo en que las preguntas planteadas en los cuestionarios previos se adecúan al contenido de las prácticas, un 56,52\% se mostraba bastante de acuerdo en que la realización de los cuestionarios previos le había ayudado a entender mejor la práctica, un 52,17\% estaba bastante de acuerdo en que había notado diferencia al enfrentarse a las prácticas los días que le había tocado hacer los cuestionarios previos respecto de los que no y un 52,18\% entendía que la realización de los cuestionarios pre y post-práctica le ayudarían a superar el examen de prácticas (Figura 2). Respecto de la valoración global de la actividad, un 56,52\% se mostró satisfecho y un 34,78\% muy satisfecho, frente al $8,78 \%$ que le resultó indiferente (Figura 2). En general, la mayoría de los estudiantes consideró útil a actividad y además resultó satisfecho con la misma.

\section{Conclusiones}

Al fomentar el repaso de los contenidos teóricos relacionados con las diferentes prácticas, utilizando para ello un entorno atractivo para el estudiante como es la plataforma MOODLE, se consigue mejorar significativamente el grado de comprensión de los conceptos prácticos. De acuerdo con nuestros resultados, al motivar a los estudiantes a responder una serie de cuestionarios alojados en MOODLE días previos a la realización de las prácticas se consigue que, al ser evaluados sobre las mismas, sus calificaciones mejoren. Teniendo en cuenta que los cuestionarios previos se diseñaron de tal forma que permitían repasar aquellos contenidos teóricos que se consideraban indispensables para el desarrollo de la práctica y a la vista de los resultados presentados, se puede concluir que esta herramienta innovadora permite impartir las clases prácticas en unas condiciones más favorables no solo para el alumno sino también para el profesor. Al encontrarnos ante unos estudiantes con una base teórica más sólida, la docencia puede orientarse más hacia el aprendizaje práctico y no tanto a un recuerdo de aquello que ya se supone impartido en las clases teóricas.

(c)) BY-NC-ND 2021, Universitat Politècnica de València

CRQJHMR, Q5 HGHपQTI 


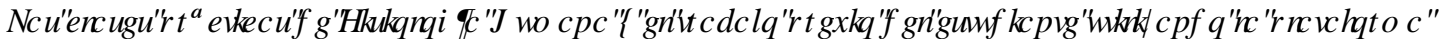 $022^{\prime}$ / ( ILXQHMXGRDDSIHGHDXФ)}

Por tanto, la presente innovación aporta una herramienta sencilla y fácil de aplicar en las aulas de prácticas de Fisiología Humana, al servicio de alumnos y profesores, cuyos efectos positivos hemos podido comprobar y que además cuenta con una gran aceptación entre los estudiantes.

\section{Ítem 1}

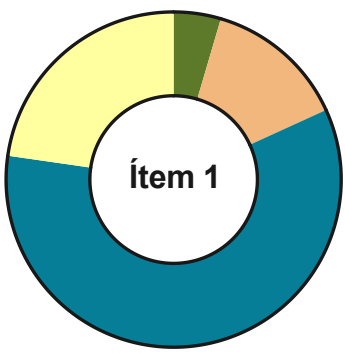

Las preguntas planteadas en los cuestionarios previos se adecúan al contenido de las prácticas

- $4.55 \% \quad$ (2) Bastante en desacuerdo

$13.64 \%$ (3) Ni de acuerdo no en desacuerdo

- 59.08\% (4) Bastante de acuerdo

$22.73 \%$ (5) Totalmente de acuerdo

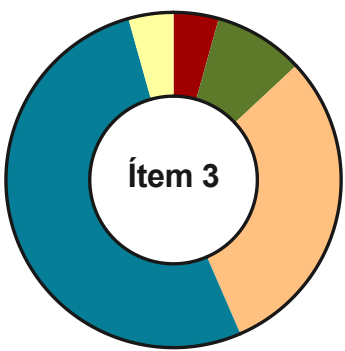

Ítem 3

He notado diferencia al enfrentarme a las prácticas los días que me había tocado hacer los cuestionarios previos respecto de los que no.
$4.35 \%$ (1) Totalmente en desacuerdo
$8.70 \%$ (2) Bastante en desacuerdo
$31.82 \%$ (3) Ni de acuerdo no en desacuerdo
- $52.17 \%$ (4) Bastante de acuerdo
$4.35 \%$ (5) Totalmente de acuerdo

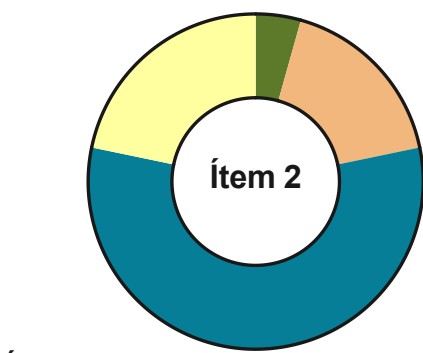

Ítem 2

La realización de los cuestionarios previos me ha ayudado a entender mejor la práctica
$4.35 \%$ (2) Bastante en desacuerdo
$17.39 \%$
$56.52 \%$
(3) Ni de acuerdo no en desacuerdo $21.74 \%$
(4) Bastante de acuerdo
(5) Totalmente de acuerdo

\section{Ítem 4}

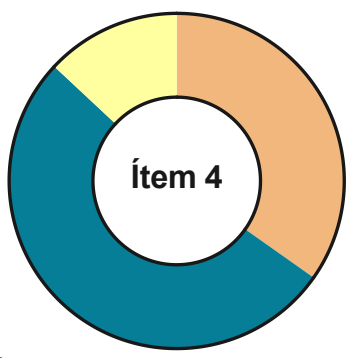

La realización de los cuestionarios pre y post-práctica me ayudarán a superar el examen de prácticas
- $52.18 \%$
(3) Ni de acuerdo no en desacuerdo
(4) Bastante de acuerdo
$13.04 \%$ (5) Totalmente de acuerdo

\section{Ítem 5}

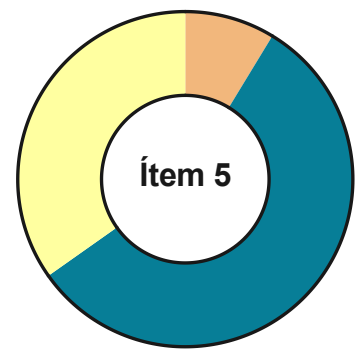

Valora la actividad del 1 al 5
$8.70 \%$
(3) Indiferente
$56.52 \%$ (4) Satisfecho
$34.78 \%$ (5) Muy satisfecho

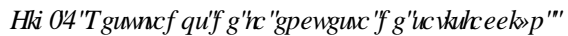




\section{Referencias}

GARCÍA, D.E. (2013). "Cómo elaborar una práctica de fisiología con un enfoque por competencias" en ,QYHMWDFlyQ HQ( GXFDFIy Q pGFD, vol. 2, issue 7, p. 168-170.

GUTIERREZ ESTEBAN, P. et al. (2011). "Buenas prácticas en el desarrollo de trabajo colaborativo en materias TIC aplicadas a la educación" en 5HILDGH\&XUFXXP पIRLP DFIy QGHOSURIHRWRR, vol. 15, issue 1, p. 179-194.

OLMOS, G. et al. (2014). "Elaboración y empleo de materiales didácticos para la mejora de la enseñanza práctica en la asignatura de Fisiología Humana en el grado de Ciencias de la Actividad Física y del Deporte. Evaluación de resultados" en 5HIUDGH8 QIYHUGDGI D6RFIHDDEGHD\&RQRFIP IHQR, vol. 11, issue 1, p. 108-128.

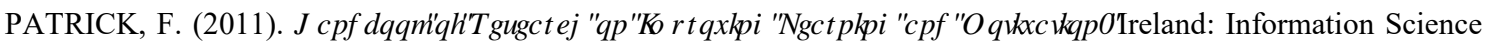
Reference.

RODRIGUEZ-IZQUIERDO, R.M. (2014). "Modelo formativo en el Espacio Europeo de Educación Superior: valoraciones de los estudiantes" en \$XOOI\$ EIHUD, vol. 42, issue 2, p. 106-113.

RUÉ, J. (2009). ( ODSUDQ] DNFIDXWQRP RHHQ( GXFDFIYQ6XSHURUIMadrid: Narcea S.A. de Ediciones.

TEJADA PONCE, Á. y LÓPEZ, M. (2012). "Nuevas metodologías docentes en los títulos de gradola literatura como recurso pedagógico colaborativo" en \$XФ) \$EIHWD, vol. 40, issue 3, p. 107-114.

ZABALZA, M. (2003-2004). “Innovación en la enseñanza universitaria” en \&RQUA URVHGXFDUMRV, vol. 6, issue 7, p. 113-136. 